\title{
Asymmetric Event-Driven Node Localization in Wireless Sensor Networks
}

\author{
Radu Stoleru, Member, IEEE, Tian He, Member, IEEE, \\ Siddhartha S. Mathiharan, Stephen M. George, and John A. Stankovic, Fellow, IEEE
}

\begin{abstract}
Localization of wireless sensor nodes has long been regarded as a problem that is difficult to solve, especially when considering characteristics of real-world environments. This paper formally describes, designs, implements, and evaluates a novel localization system called Spotlight. The system uses spatiotemporal properties of well-controlled events in the network, light in this case, to obtain locations of sensor nodes. Performance of the system is evaluated through deployments of Mica2 and XSM motes in an outdoor environment, where $20 \mathrm{~cm}$ localization error is achieved. A sensor network consisting of any number of nodes deployed in a $2,500 \mathrm{~m}^{2}$ area can be localized in under 10 minutes. Submeter localization error in an outdoor environment is made possible without equipping the wireless sensor nodes with specialized ranging hardware.
\end{abstract}

Index Terms-Wireless sensor networks, node localization, asymmetric function.

\section{INTRODUCTION}

$\mathrm{L}$ OCALIZATION - finding the position of individual sensor - nodes-remains one of the most difficult research challenges. Practical solutions involving reasonable power, computation and monetary costs do not exist. Since many emerging applications based on networked sensors require location awareness, a node must be able to find its location.

Various approaches have been proposed in the literature [1], [2], [3], [4], [5], [6]; however, it is still not clear how these solutions can be practically and economically deployed. An on-board GPS [5] is a typical high-end solution requiring sophisticated hardware. However, power and cost constraints for tiny sensor nodes preclude this as a viable solution. Other hardware approaches require per node devices to perform ranging but present two difficulties. First, given typical form factor and power constraints, the effective range of such devices is very limited. For example, the effective range of the ultrasonic transducers is less than 2 meters when the sender and receiver are not facing each other [7]. Second, since the locations of most sensor nodes are fixed, it is not cost-effective to equip each sensor with special circuitry for one-time localization. To overcome these limitations, range-free localization schemes have been proposed. Most such schemes estimate the location of sensor nodes by exploiting radio connectivity information among neighboring nodes. These approaches eliminate the

- R. Stoleru, S.S. Mathiharan, and S.M. George are with the Department of Computer Science and Engineering, Texas A\&M University, College Station, TX 77845. E-mail: \{stoleru, sidsan, smgeorge\}@cse.tamu.edu.

- T. He is with the Department of Computer Science and Engineering, University of Minnesota, Minneapolis, MN 55455.

E-mail: tianhe@cse.umn.edu.

- J.A. Stankovic is with the Department of Computer Science, University of Virginia, Charlottesville,VA 22903. E-mail: stankovic@cs.virginia.edu.

Manuscript received 14 Feb. 2010; revised 23 June 2010; accepted 10 Aug. 2010; published online 24 Aug. 2011.

Recommended for acceptance by Y. Liu.

For information on obtaining reprints of this article, please send e-mail to: tpds@computer.org, and reference IEEECS Log Number TPDS-2010-02-0101. Digital Object Identifier no. 10.1109/TPDS.2011.227. need for specialized hardware at the cost of less accurate localization. However, since radio propagation characteristics vary over time and are environment dependent, range-free localization schemes incur high calibration costs to correct for this variance.

This paper proposes Spotlight, a localization system that delivers high-location estimation accuracy at low cost. Using an asymmetric architecture with all sophisticated hardware and computation in a single device, Spotlight offers various techniques that allow users to balance time and accuracy to obtain results tailored to requirements. In all cases, the only limiting factor is the total size of the sensor field. Any number of sensors may be localized within a covered area at no additional cost, making Spotlight suitable for large-scale deployments.

\section{Spotlight System Design}

The core concept of the Spotlight localization system is the generation of controlled events detectable by deployed sensor nodes. Events like light and sound, with wellcharacterized spatiotemporal properties and detectable with simple sensing hardware, perform well in this system. By measuring a sensor node's detection time of a generated event, a spatial relationship between the sensor node and the event generator can be inferred.

A typical military sensor network serves as an example. Wireless sensor nodes are deployed from an unmanned aerial vehicle. After deployment, the sensor nodes selforganize into a network and synchronize clocks. A second aerial vehicle, with accurate knowledge of its position (e.g., GPS) and orientation (three translation and three rigidbody-rotation degrees of freedom) uses an onboard Spotlight device to generate light events in the sensor field. Sensor nodes detect the events and report detection time to designated nodes within the network. The Spotlight system collects this data and computes the 3D locations of all sensors reporting event detection. Availability of 


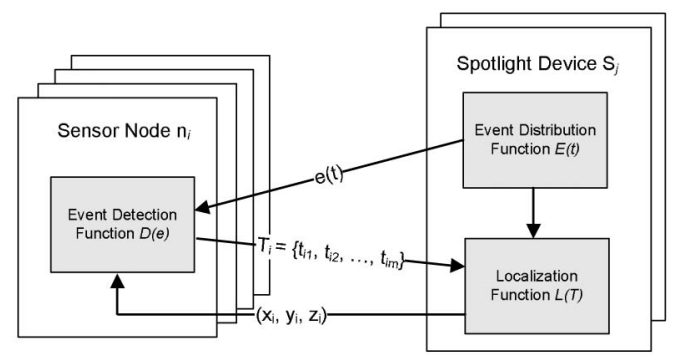

Fig. 1. Spotlight system architecture, depicting the Event Detection function $E(t)$, implemented by sensor nodes and EDFs $D(e)$, implemented by the Spotlight device.

geographic data, like terrain elevations, improves the Spotlight system's accuracy.

The following sections formally define the localization problem in the Spotlight system and describe and analyze different types of events (primitives, as well as hybrid solutions) that can be generated.

\subsection{Definitions and Problem Formulation}

Assume a space $A \subset R^{3}$ containing all sensor nodes, where each node $n_{i}$ is positioned at $P_{i}\left(x_{i}, y_{i}, z_{i}\right)$. To obtain $P_{i}\left(x_{i}, y_{i}, z_{i}\right)$, the Spotlight localization system, as depicted in Fig. 1, supports three main functions: the Event Distribution Function (EDF) $E(t)$, the Event Detection Function $D(e)$, and the Localization Function $L\left(T_{i}\right)$. More formally, an event is defined as

Definition 1. An event $e(t, P)$ is a detectable phenomenon (e.g., light, heat, smoke, sound) that occurs at time $t$ and at point $P \in A$.

As shown in Fig. 1, Event Detection $D(e)$ is supported by the sensor nodes. It determines whether an external event happens or not (it can be implemented through either simple threshold-based detection algorithms or using more advanced digital signal processing techniques). Localization Functions $L\left(T_{i}\right)$ are implemented by Spotlight devices and typically consist of an aggregation algorithm which calculates the intersection of multiple sets of events. Event Distribution $E(t)$ describes the distribution of events over time and is present on one or more Spotlight devices. As the core technique of the Spotlight system, it is much more sophisticated than the other two functions. Since $E(t)$ is computed by the Spotlight device, hardware requirements for the sensor nodes remain minimal. Substantial algorithmic changes can be made without requiring updates on deployed sensor nodes. The three functions and associated elements are formally defined as

Definition 2. For a given event e, the Event Detection Function $D(e)$ defines a binary detection algorithm

$$
D(e)= \begin{cases}\text { true } & \text { if event } \text { e is detected } \\ \text { false } & \text { if event } \text { is not detected. }\end{cases}
$$

Definition 3. Let $S_{j}(x, y, z)$ be the coordinates of the Spotlight device, and $d_{i}$ be the direction cosine of the line joining the Spotlight device $S_{j}$ and a sensor $n_{i}$. The EDF E $(t)$ defines a set

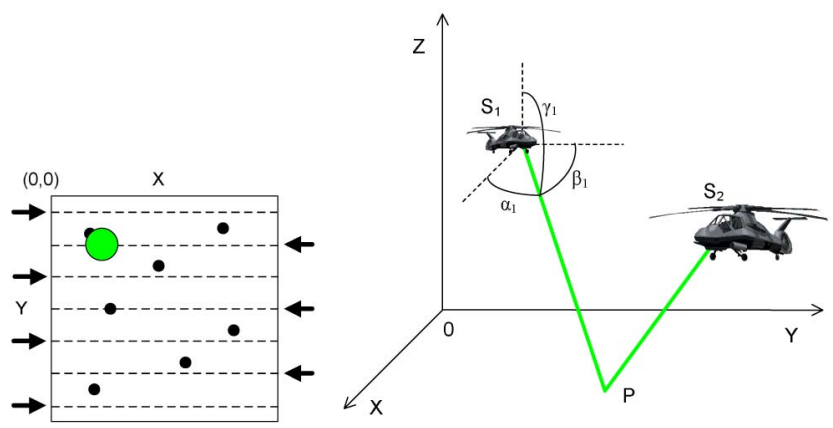

(a)

(b)

Fig. 2. The Point Scan EDF: (a) an idealized view; (b) a realistic view, in an unknown terrain.

of points $P$ that describe the intersection of $d_{i}$ and $A$. These are the points where the event can be detected by a node

$$
\begin{aligned}
E(t) & =\{P \mid P \in A \text { and } D(e(t, P))=\text { true and } \\
P & \left.=S_{j}+c \times d_{i} \text { where } c \in Z\right\} .
\end{aligned}
$$

Definition 4. Let $T_{i}=\left\{t_{i 1}, t_{i 2}, \ldots, t_{i m}\right\}$, the set of $m$ time stamps of events detected by node $n_{i}$. Localization Function $L\left(T_{i}\right)$ defines an algorithm with input $T_{i}$

$$
L\left(T_{i}\right)=\bigcap_{t \in T_{i}} E(t) .
$$

With the support of these three functions, the localization process proceeds as follows:

- A Spotlight device distributes events in the space $A$.

- During event distribution, sensor nodes record the time sequence $T_{i}=\left\{t_{i 1}, t_{i 2}, \ldots, t_{i m}\right\}$ at which they detect the events.

- After event distribution, each sensor node sends the detection time sequence $T_{i}$ to the Spotlight device.

- The Spotlight device estimates the location of a sensor node $n_{i}$, using $T_{i}$ and the known $E(t)$ function.

The EDF $E(t)$ may be tuned to distribute events optimally based on limitations imposed by sensor capabilities, limitations of the platform transporting the Spotlight system, limitations imposed by terrain, and availability of detailed geographic information. The Point Scan, Line Scan, and Area Cover Event Functions each illustrate basic functionality of the Spotlight localization system. Each of these designs is evaluated in three scenarios: 1) the terrain is known or assumed to be flat; 2) terrain information is available; and 3) the terrain is unknown.

\subsection{Point Scan Event Distribution Function}

Some devices [8] can create events of very small size when compared with the deployment area. Such "point" events are described by the Point Scan EDF. Fig. 2a depicts the Point Scan EDF, where a Spotlight device generates point events (e.g., light spots) in an $A \in R^{2}$ area along the $x$-axis. Assuming that the scanning speed is a constant $s$, that the deployment area is $A=l \times l$, and that the radius of the event is $r$, the EDF is given by 


$$
\begin{gathered}
E(t)=\{P \mid P(x, y) \in A \text { and } x=(s t) \bmod (l) \text { and } \\
y=\lfloor s t / l\rfloor r\} .
\end{gathered}
$$

The resulting localization function is

$$
L\left(T_{i}\right)=E\left(t_{i 1}\right)=\left\{\left(s t_{i 1}\right) \bmod (l),\left\lfloor s t_{i 1} / l\right\rfloor r\right\},
$$

where $D\left(e\left(t_{i 1}, P_{i}\right)\right)=$ true for node $n_{i}$ positioned at $P_{i} . t_{i 1}$ is the time stamp of the event detected by a node.

The scenario depicted in Fig. 2a is simplified. In a real deployment, the locations of sensor nodes need to be computed from the known location and orientation of the Spotlight device, and, if available, from information about the deployment area. The time stamps $T_{i}$ of events detected by nodes provide, in essence, enough information to calculate a line between the sensor node and the Spotlight device. The calculation of this line is aided by the fact that the Spotlight device knows its location and the EDF parameters: angles $\alpha_{1}, \beta_{1}$, and $\gamma_{1}$, as shown in Fig. $2 \mathrm{~b}$, at different times. Hence, given a time stamp, a node can reside anywhere along a line in $A$. To be able to compute a unique location for a node, we must either assume that the map of the deployment area is known, or have the Point Scan EDF created from multiple locations. Two scenarios, one in which the map of the deployment area is known and the other in which it is unknown, are formally described in the remaining part of this section.

For known terrain. Let $S_{1}=\left(x_{1}, y_{1}, z_{1}\right)^{T}$ be the coordinates of the Spotlight device. Let $\alpha_{1}, \beta_{1}$, and $\gamma_{1}$ be the angles made with the $X-, Y$-, and $Z$-axes, respectively, as shown in Fig. $2 b$. Then, the equation of the line from Spotlight device to the terrain is given by

$$
\left(\begin{array}{l}
x \\
y \\
z
\end{array}\right)=\left(\begin{array}{l}
x_{1} \\
y_{1} \\
z_{1}
\end{array}\right)+k\left(\begin{array}{c}
\cos \alpha_{1} \\
\cos \beta_{1} \\
\cos \gamma_{1}
\end{array}\right) .
$$

Let the terrain be represented using a Height Map (HM). An $\mathrm{HM}$ is described by a 3 -tuple $\left(x_{i}, y_{i}, z_{i}\right)$. When the Spotlight beam intersects the terrain at multiple points, the real intersection has the highest $z$ value. Hence, the values of $x$ and $y$ can be mapped for various values of $z$ to see if the resulting point belongs to the $\mathrm{HM}$

$$
\begin{gathered}
k=\frac{x-x_{1}}{\cos \alpha_{1}}=\frac{y-y_{1}}{\cos \beta_{1}}=\frac{z-z_{1}}{\cos \gamma_{1}} \\
\left(\begin{array}{l}
x \\
y
\end{array}\right)=\frac{z-z_{1}}{\cos \gamma_{1}}\left(\begin{array}{c}
\cos \alpha_{1} \\
\cos \beta_{1}
\end{array}\right)+\left(\begin{array}{l}
x_{1} \\
y_{1}
\end{array}\right) .
\end{gathered}
$$

Substituting different values for $z$ allows the discovery of the maximum $z$ such that $x, y$, and $z$ belong to HM

$$
\begin{aligned}
\left(\begin{array}{l}
x \\
y \\
z
\end{array}\right)= & \max _{z}\left\{\left(\begin{array}{l}
x \\
y
\end{array}\right)=\frac{z-z_{1}}{\cos \gamma_{1}}\left(\begin{array}{c}
\cos \alpha_{1} \\
\cos \beta_{1}
\end{array}\right)\right. \\
& \left.+\left(\begin{array}{l}
x_{1} \\
y_{1}
\end{array}\right) \text { and }\left(\begin{array}{l}
x \\
y \\
z
\end{array}\right) \in H M\right\} .
\end{aligned}
$$

The resulting localization function is

$$
\begin{aligned}
L\left(T_{i}\right)=E\left(t_{i 1}\right)= & \max _{z}\left\{\left(\begin{array}{l}
x \\
y
\end{array}\right)=\frac{z-z_{1}}{\cos \gamma_{1}}\left(\begin{array}{c}
\cos \alpha_{1} \\
\cos \beta_{1}
\end{array}\right)\right. \\
& \left.+\left(\begin{array}{l}
x_{1} \\
y_{1}
\end{array}\right) \text { and }\left(\begin{array}{l}
x \\
y \\
z
\end{array}\right) \in H M\right\},
\end{aligned}
$$

where $\alpha_{1}, \beta_{1}$, and $\gamma_{1}$ are functions of time and are calculated using the time stamp $t_{i 1}$ received.

For unknown terrain. If the map of the deployment area is not known, the Spotlight device needs to create the EDF $E(t)$ from two different locations, $S_{1}$ and $S_{2}$, as depicted in Fig. 2b. If $V_{1}$ and $V_{2}$ are the direction vectors for each of the beams created from the two Spotlight device locations, then the directions of the Point Scan EDF events create from $S_{1}$ and $S_{2}$ can be formally expressed as

$$
l_{1}=S_{1}+c_{1} V_{1} \quad \text { and } \quad l_{2}=S_{2}+c_{2} V_{2},
$$

where $c_{1}$ and $c_{2}$ are parameters. Solving for the intersection of the two beams, i.e., $l_{1}=l_{2}$, we obtain

$$
c_{1} V_{1}=\left(S_{2}-S_{1}\right)+c_{2} V_{2} .
$$

After taking the cross product of both sides with $V_{2}$

$$
c_{1}\left(V_{1} \times V_{2}\right)=\left(S_{2}-S_{1}\right) \times V_{2} .
$$

Substituting for $c_{1}$ in (5), we obtain $P$, the vector representing the position of the sensor

$$
P=S_{1}+\left|\frac{\left(S_{2}-S_{1}\right) \times V_{2}}{\left(V_{1} \times V_{2}\right)}\right| V_{2},
$$

where

$$
\begin{aligned}
& S_{1}=\left(x_{1}, y_{1}, z_{1}\right), S_{2}=\left(x_{2}, y_{2}, z_{2}\right), \\
& V_{1}=\left(\cos \alpha_{1}, \cos \beta_{1}, \cos \gamma_{1}\right)^{T},
\end{aligned}
$$

and $V_{2}=\left(\cos \alpha_{2}, \cos \beta_{2}, \cos \gamma_{2}\right)^{T}$. It is important to remember that $V_{1}$ and $V_{2}$, the directional cosines of the line between Spotlight device and sensor, are a function of time. As shown, six parameters (three rotation $-\alpha_{i}, \beta_{i}$, and $\gamma_{i}$, the angles made with the $X, Y$, and $Z$-axes, respectively, and three translation $-x_{i}, y_{i}$, and $z_{i}$, the coordinates of the Spotlight device) are used to find the location $P$ of the sensor node.

The EDF $E(t)$ for the Spotlight devices $S_{1}$ and $S_{2}$ are then given by

$$
\begin{aligned}
& E_{S_{1}}\left(t_{i 1}\right)=\left\{P \mid P \in A \text { and } P=S_{1}+c V_{1}\right\} \\
& E_{S_{2}}\left(t_{i 2}\right)=\left\{P \mid P \in A \text { and } P=S_{2}+c V_{2}\right\} .
\end{aligned}
$$

The localization function $L\left(T_{i}\right)$ is then

$$
L\left(T_{i}\right)=E_{S_{1}}\left(t_{i 1}\right) \bigcap E_{S_{2}}\left(t_{i 2}\right)=S_{1}+\left|\frac{\left(S_{2}-S_{1}\right) \times V_{2}}{\left(V_{1} \times V_{2}\right)}\right| V_{2},
$$

where $V_{1}$ and $V_{2}$ are a function of time and are calculated using the time stamps $t_{i 1}$ and $t_{i 2}$ received, and $D\left(e\left(t_{i 1}, P_{i}\right)\right)=$ true and $D\left(e\left(t_{i 2}, P_{i}\right)\right)=$ true for node $n_{i}$ positioned at $P_{i}$.

\subsection{Line Scan Event Distribution Function}

Some devices, like diode lasers [8], can generate an entire line of events simultaneously. With these devices, it is 


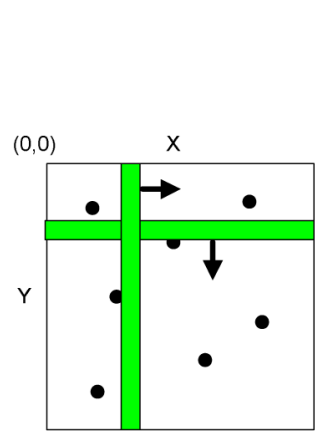

(a)

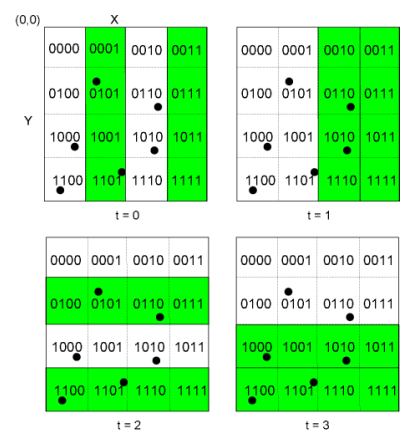

(b)
Fig. 3. (a) Line Scan EDF: an idealized view; (b) Area Cover EDF with events covering the shaded areas.

possible to support the Line Scan EDF. The main idea of Line Scan EDF is depicted in Fig. 3a, where a Spotlight device simultaneously generates vertical and horizontal scans of the deployment area.

In this scenario, the sensor nodes are assumed to populate a plane $\left(A=[l \times l] \subset R^{2}\right)$. The scanning speed is $s$ and the set of time stamps of events detected by a node $i$ is $T_{i}=\left\{t_{i 1}, t_{i 2}\right\}$. The Line Scan EDF is defined as

$$
\begin{aligned}
& E(t)=\{P \mid P \in A \text { and } P=(t s, k) \text { where } k \in[0, l]\} \\
& E(t)=\{P \mid P \in A \text { and } P=(k, t s) \text { where } k \in[0, l]\},
\end{aligned}
$$

for $t \in[0, l / s]$ and $t \in[l / s, 2 l / s]$, respectively. A node is localized by calculating the intersection of the two event lines. More formally

$$
L\left(T_{i}\right)=E\left(t_{i 1}\right) \bigcap E\left(t_{i 2}\right),
$$

where $D\left(e\left(t_{i 1}, P_{i}\right)\right)=$ true, $D\left(e\left(t_{i 2}, P_{i}\right)\right)=$ true for node $n_{i}$ positioned at $P_{i}$. The scenario depicted in Fig. 3a is, however, simplified. In a real deployment, the locations of sensor nodes need to be computed from the known location and orientation of the Spotlight device, and, if available, from information about the deployment area. For the Line Scan EDF, the time stamps $T_{i}$ of events detected by nodes provide enough information to calculate the plane formed by the location of the Spotlight device and the line scan. As was the case in Point Scan EDF, we can distinguish between two scenarios: one in which the map of the deployment area is known and the other in which it is unknown. These scenarios are formally described in Supplemental material, which can be found on the Computer Society Digital Library at http://doi.ieeecomputersociety.org/10.1109/ TPDS.2011.227, Section 1.

\subsection{Area Cover Event Distribution Function}

The Point Scan and Line Scan functions require precise tracking of the event generator orientation. Area Cover EDF lessens the precision required by using devices, like light projectors, to generate events that cover an area.

Area Cover EDF can be illustrated with a simple example. As shown in Fig. $3 b$, the plane $A$ is divided in 16 sections and each section $S_{k}$ is assigned a unique code $k$. The Spotlight device distributes events according to these codes: at time $j$, a section $S_{k}$ is covered by an event (illuminated, in the case of a visible light event) if $j$ th bit of $k$ is 1 . A node residing anywhere in a section $S_{k}$ is localized to that section's center. For example, nodes within section " 1010 " detect the events at time $T=\{1,3\}$. $S_{k}$ for any sensor in the area covered by the event generator can be determined at $t=4$.

In the Area Cover EDF, the space $A$ is partitioned and each section is assigned a unique binary identifier, or code. The Area Cover EDF is then formally defined as

$$
B I T(k, j)=\left\{\begin{array}{cl}
\text { true } & \text { if } j \text { th bit of } k \text { is } 1 \\
\text { false } & \text { if } j \text { th bit of } k \text { is } 0,
\end{array}\right.
$$

and the corresponding localization algorithm is

$$
\begin{aligned}
L\left(T_{i}\right)= & \left\{p \mid p=C O G\left(S_{k}\right) \text { and }\left(B I T(k, t)=\text { true if } t \in T_{i}\right)\right. \\
& \text { and } \left.\left(B I T(k, t)=\text { false if } t \in T^{\prime}-T_{i}\right)\right\},
\end{aligned}
$$

where $\operatorname{COG}\left(S_{k}\right)$ denotes the center of gravity of $S_{k}$.

Based on the set of time stamps received, a node can be localized to the center of a section. In cases where the plane of the sensors is unknown, the lines to the four corners of each section can be calculated and the position localized to the center of the square formed. Using mechanisms similar to those used in Point Scan and Line Scan, the equation of the line to the localized point can be used to find the sensor's position in unknown and known terrains. Section 2 of the Supplemental material, which can be found on the Computer Society Digital Library, analyzes the impact of error correction, code placement, and grid size on localization accuracy.

\subsection{Hybrid and Optimal EDFs}

The three EDFs vary in overhead and energy consumption. A hybrid solution attempts to leverage strengths of more than one function to optimize localization while minimizing total cost. Hybrid Distribution Functions are a combination of Line Scan and Area Cover or a combination of Area Covers using different event sizes. These combined distribution functions are optimized using criteria like average localization error and total localization time. Uniform or nonuniform sensor node distribution may be assumed across different scan regions. Supplemental material, which can be found on the Computer Society Digital Library, Section 3, examines the impact of density assumptions in hybrid EDFs.

\subsection{Event Distribution Function Analysis}

Point Scan, Line Scan, and Area Cover EDFs all localize sensor nodes. However, they vary in localization time, communication overhead, and energy consumed (defined as Event Overhead). Assume that all sensor nodes are located in a square with edge size $D$, and that the Spotlight device can generate $N$ events (e.g., Point, Line, and Area Cover events) every second, and that the maximum tolerable localization error is $r$. Table 1 compares the execution cost of the three techniques.

Table 1 indicates that the Event Overhead for the Point Scan method is the smallest-it requires a one-time coverage of the area, hence the $D^{2}$. However, the Point Scan takes a much longer time than the Area Cover technique, which finishes in $\log _{r} D$ seconds. The Line Scan method increases Event Overhead but decreases localization time. By doubling 
TABLE 1

Execution Cost Comparison Criterion

\begin{tabular}{|l|c|c|c|}
\hline Criterion & Point Scan & Line Scan & Area Cover \\
\hline Localization Time & $\left(D^{2} / r^{2}\right) / N$ & $(2 D / r) / N$ & $\log _{r} D / N$ \\
\hline \# Event Detections & 1 & 2 & $\log _{r} D$ \\
\hline Event Overhead & $D^{2}$ & $2 D^{2}$ & $D^{2} \log _{r} D / 2$ \\
\hline
\end{tabular}

the Event Overhead, the Line Scan method takes only $r / 2 D$ percentage of time to complete, when compared with the Point Scan method. Similarly, it can be observed that the execution costs are independent of the number of nodes to be localized. In terms of power usage, the ratio of Event Overhead per unit time can be used to estimate power requirements for the Spotlight device. This ratio is constant for the Point Scan $\left(r^{2} N\right)$ while it grows linearly with area for the Area Cover $\left(D^{2} N / 2\right)$. If the deployment area is very large, the use of the Area Cover EDF is prohibitively expensive, if not impossible. For practical purposes, Area Cover is a viable solution for small to medium size networks, while the Line Scan works well for large networks.

\section{SYSTEM IMPLEMENTATION}

Two Spotlight systems were implemented. These implementations enabled investigation of the full spectrum of Event Distribution techniques.

The first implementation, called $\mu$ Spotlight, had a short range $(10-20 \mathrm{~m})$. However, its ability to generate the entire spectrum of EDFs made it very useful. This scaled-down implementation was used to investigate capabilities of the Spotlight system and to tune performance. It was not intended to represent a full solution.

The second implementation, the Spotlight system, had a much longer range (as far as 6,500 m), but was limited in the types of EDFs generated. The goal of this implementation was to show the Spotlight system working in a real, outdoor environment, and to show correlations with the experimental results obtained from the $\mu$ Spotlight system implementation.

Supplemental material, which can be found on the Computer Society Digital Library, Section 4, provides additional details about hardware, implemented event detection and localization functions, time synchronization, and limitations and alternatives to the use of visible light.

\section{Performance Evaluation}

This section presents performance evaluations of Spotlight systems using the three EDFs, i.e., Point Scan, Line Scan, and Area Cover, described in Section 2. Metrics of interest in the experimental evaluation were

- Localization error: the distance between real and Spotlight-estimated locations.

- Localization duration: the time span between the first and last event.

- Localization range: the maximum distance between the Spotlight device and the sensor nodes.

- Localization bias: used to investigate the effectiveness of the calibration procedure. If, for example, all

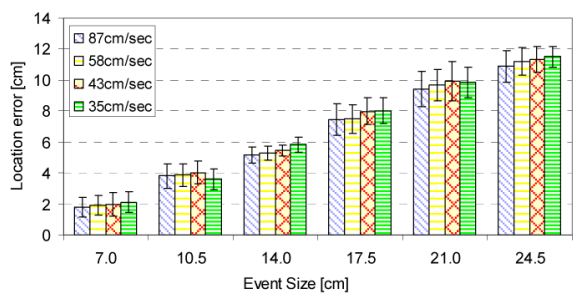

(a)

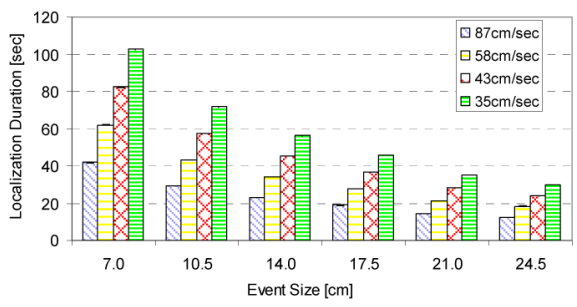

(b)

Fig. 4. (a) Localization Error versus Event Size for Point Scan EDF; (b) Localization Duration versus Event Size for Point Scan EDF.

computed locations are biased in the west direction, a calibration factor can be used to compensate.

Supplemental material, which can be found on the Computer Society Digital Library, Section 5, provides additional details on an additional metric: a localization cost function. The parameters that were varied during the performance evaluation of the system include: the type of EDF (Point, Line, and Area), the size of the event, the duration of the event (for Area Cover), the scanning speed, the power of the laser, and the range. The $\mu$ Spotlight system was evaluated in an indoor environment using 20 motes on a field of approximately $2.5 \times 6 \mathrm{~m}^{2}$. The Spotlight system was evaluated in a sports stadium with 20 nodes deployed to one end of the field. Events were generated at a range of $170 \mathrm{~m}$ from the seating areas.

\subsection{Point Scan $-\mu$ Spotlight System}

Sources of localization error using the Point Scan EDF were investigated by varying event size and scanning speed. Notably, varying the scanning speed between 35 and $87 \mathrm{~cm} / \mathrm{sec}$ had minor influence on accuracy but the size of the event had dramatic effect.

Localization error attributed to event size varied from 2-8 cm (Fig. 4a). This dependence is explained by the Event Detection algorithm: the first detection above a threshold sets the time stamp for the event. The duration of the localization scheme is directly proportional to scanning speed, as shown in Fig. 4b. The dependency of localization duration on event size and scanning speed is natural. Bigger events allow a reduction in the total duration of up to 70 percent.

The trade-off between localization accuracy and time is interesting. Accuracy is normally paramount. However, scenarios requiring stealthiness or where the mobile platform performs various tasks may consider time paramount.

\subsection{Line Scan- $\mu$ Spotlight System}

The dependency of the localization error and duration on event size and scanning speed was investigated for the Line Scan EDF. Fig. 5a plots localization error for different event 


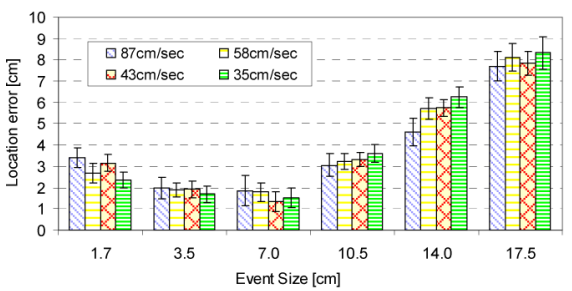

(a)

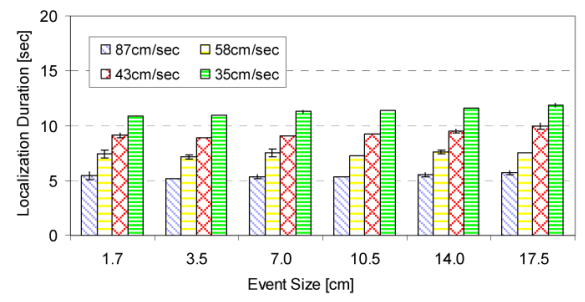

(b)

Fig. 5. (a) Localization Error versus Event Size for Line Scan EDF; (b) Localization Duration versus Event Size for Line Scan EDF.

sizes. It is interesting to observe the dependency (concave shape) of the localization error versus event size. It is notable that a similar dependency did not arise in the case of Point Scan EDF.

The explanation for this dependency is the existence of bias in the location estimation (a bias factor was introduced in order to best estimate the central point of events that have a large size; a large event is detected when its edge triggers the sensor). Our location estimation used a single bias value for all experiments, regardless of event size. Fig. 5a shows that the bias factor was optimal for an event size of approximately $7 \mathrm{~cm}$. For events smaller and larger than this, the bias factor was too large, and too small, respectively. Thus, it introduced bias in the position estimation.

The same dependency was not observed in the case of the Point Scan EDF, because the experiment did not consider event sizes below $7 \mathrm{~cm}$, due to the long time it would have taken to scan the entire field.

The results for localization duration as a function of event size are shown in Fig. 5b. As shown, localization duration is directly proportional to scanning speed. Event size has a smaller influence on localization duration. The average localization duration of approximately $10 \mathrm{sec}$ is much shorter than the duration obtained in the Point Scan experiment.

Experiments with Line Scan EDF revealed evidence of a bias in location estimation. The estimated locations for all sensor nodes exhibited different biases for different event sizes. For example, for an event size of $17.5 \mathrm{~cm}$, the estimated

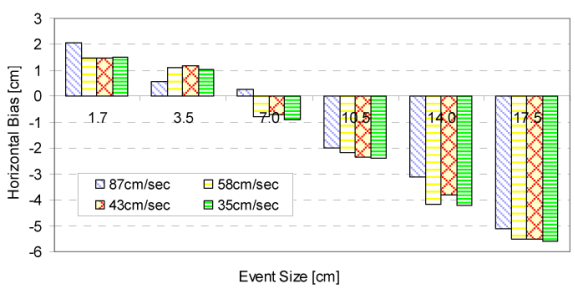

(a)

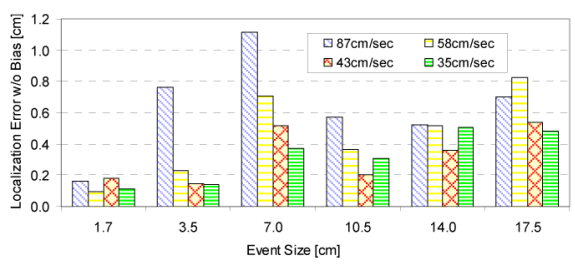

(b)

Fig. 6. (a) Position Estimation Bias for Line Scan EDF; (b) Position Estimation w/o Bias (ideal) for Line Scan EDF.

location for sensor nodes was to the upper left size of the actual location. This was equivalent to an "early" detection, since scanning was done from left to right and from top to bottom. Scanning speed did not influence bias. To gain insight, additional data analysis was done. Fig. 6a shows bias in the horizontal direction, for different event sizes. Other analysis revealed an almost identical vertical bias.

Fig. 6a shows that the smallest observed bias, and hence the most accurate positioning, was for an event of size $7 \mathrm{~cm}$. These results are consistent with the observed localization error, shown in Fig. 5a.

Observed bias (Fig. 6a) was used to adjust the measured localization error in Fig. 5a. The results of an ideal case of Spotlight Localization system with Line Scan EDF are shown in Fig. 6b. The errors are remarkably small, varying between 0.1 and $0.8 \mathrm{~cm}$, with a general trend of higher localization errors for larger event sizes.

\subsection{Area Cover- $\mu$ Spotlight System}

This experiment investigated how the number of bits used to quantify the sensor field affected localization accuracy. The first experiment did not use error correcting codes. The results are shown in Fig. 7a.

The experiments revealed a high degree of accuracy, with localization errors on the order of $0.3-0.6 \mathrm{~cm}$. Variance in the error was observed during the experiment. In the scenario where 12 bits were used, while the average error was very small, incorrect event detection generated a larger than expected error in a few cases. Experimental results, presented

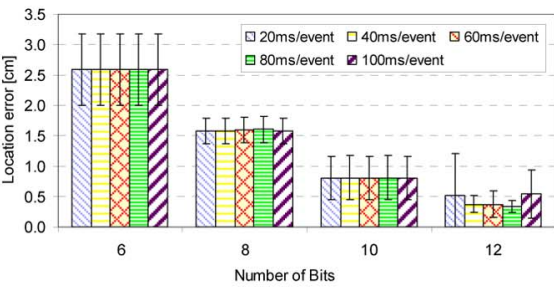

(a)

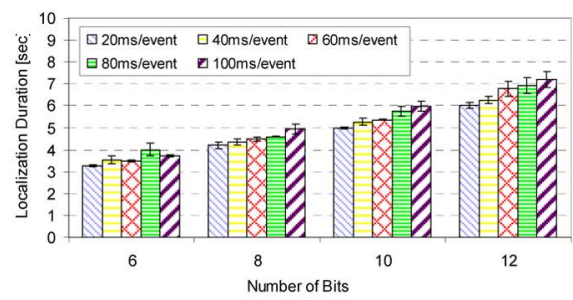

(b)

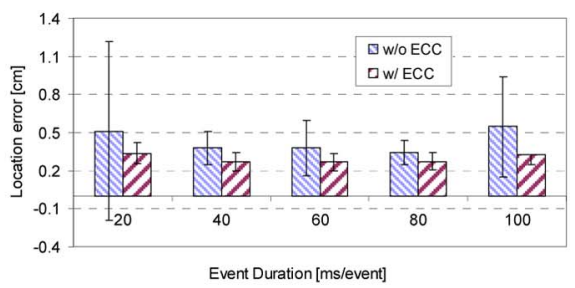

(c)

Fig. 7. (a) Localization Error versus Event Size for Area Cover EDF; (b) Localization Duration versus Event Size for Area Cover EDF; (c) Localization Error w/ and w/o Error Correction. 


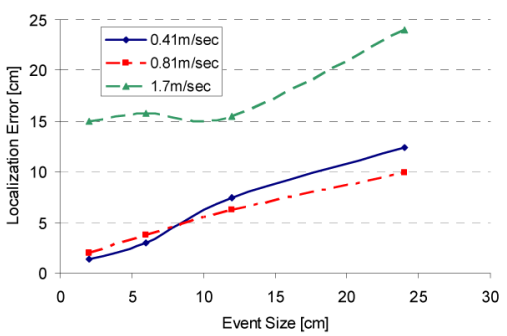

(a)

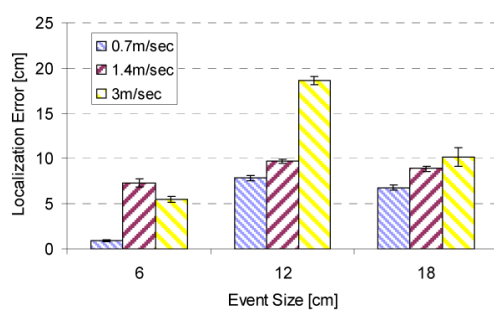

(b)

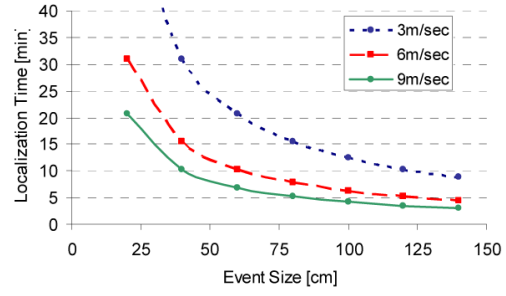

(c)

Fig. 8. (a) Localization Error versus Event Size for Spotlight at 46 m; (b) Localization Error versus Event Size for Spotlight at 170 m; (c) Localization Time versus Event Size for Spotlight.

in Fig. 7a, emphasize the need for error correction of the bit patterns observed and reported by the sensor nodes.

The localization duration results are shown in Fig. $7 \mathrm{~b}$. Duration is directly proportional to the number of bits used, with total durations ranging from $3 \mathrm{sec}$, for the least accurate method, to 6-7 sec for the most accurate. The duration of an event had little influence on the total localization time, when considering the same scenario (same number of bits for the code).

Two problematic scenarios occurred where 12-bit codes produced errors larger than the event size (Fig. 7a). These were due to detection errors and were further explored using an extended Golay $(24,12)$ error correction mechanism.

The experimental results depicted in Fig. 7c show consistent accuracy. The scenario without error correction codes is the same 12-bit code scenario shown in Fig. 7a. Only the 12-bit scenario was investigated due to its match with the 12-bit data required by the Golay encoding scheme (extended Golay producing 24-bit codewords).

\subsection{Point Scan-Spotlight System}

Evaluation of the Spotlight system was conducted using a range of experiments in a football stadium. Available hardware allowed evaluation of the Point Scan EDF system and provided insight into the performance of the system at different ranges. Figs. $8 \mathrm{a}$ and $8 \mathrm{~b}$ plot localization error versus event size at two different ranges: 46 and $170 \mathrm{~m}$. Fig. 8a reveals the extent of localization errors. The Spotlight system typically achieved localization errors of a few centimeters, normally only possible in range-based localization schemes [9]. The observed dependency on the size of the event is similar to the one observed in the $\mu$ Spotlight system evaluation. This similarity corroborated the $\mu$ Spotlight system as a viable, small-scale, inexpensive alternative for investigating complex EDFs.

In experiments performed over longer distances, the average localization error remained very small, with typical errors in the order of $5-10 \mathrm{~cm}$ (Fig. 8b). At distances of approximately $100 \mathrm{~m}$, reliable, reproducible localization was produced across a range of experiments. In many cases, observed localization errors were simply offsets of real locations. Since the same phenomenon was observed when experimenting with the $\mu$ Spotlight system, it is possible that autocalibration may further reduce localization error.

The time required for localization by the Spotlight system using a Point Scan EDF is given by: $t=(L l) /\left(s E_{s}\right)$, where $L$ and $l$ are dimensions of the sensor network field, $s$ is the scanning speed, and $E_{s}$ is the size of the event. Fig. 8c shows the time for localizing a sensor network deployed in an area of size of a football field using the Spotlight system. Message propagation time from the sensor nodes to the Spotlight device is ignored.

From Fig. 8c, it can be observed that the cost of obtaining very small localization errors is prohibitively expensive in terms of time in the case of the Point Scan. When localization errors of up to $1 \mathrm{~m}$ are tolerable, localization duration can be as low as 4 minutes. Localization durations of 5-10 minutes, and localization errors of $1 \mathrm{~m}$ are currently state of art in the realm of range-free localization schemes.

\section{Related Work}

Localization can be grossly divided into two categories: range-based and range-free localization solutions.

Range-based localization. Range-based solutions vary from simplistic, inaccurate RSSI-based algorithms to complex, highly accurate integrated hardware. RSSI is perhaps the most frequently studied ranging alternative. RADAR [10] uses RSSI to build a centralized repository of signal strengths at various positions with respect to a set of beacons. Mobile user locations are estimated within meters. Similarly, MoteTrack [11] distributes reference RSSI values to beacons. Solutions using RSSI that do not require static beacons have also been proposed [12], [3], [13], [7], [14]. Each uses mobile beacons but applies different algorithms to infer location. Priyantha et al. [7] propose MAL, where a node moves strategically to assist with range measurements until distance constraints generate a rigid graph. Pathirana et al. [13] formulate the problem as a nonlinear dynamic system and solve it with a Robust Extended Kalman Filter. More precise ranging techniques, typically using additional hardware and reduced coverage, are addressed by Cricket [9], AHLoS [6], and [15]. Savvides and colleagues offer a camera-based ranging scheme [16] where two or more cameras collaborate to localize nodes in their mutual fields of view.

Range-free localization. Range-free approaches offer an alternative to the challenges and costs imposed by rangebased schemes. These approaches localize by leveraging easily detectable, countable phenomena in the environment including hop counts to neighbors and the arrival and departure of emitters in the area.

Bulusu et al. [1] locate nodes to the centroid of local beacons. Schuhmann et al. [17] improve location estimates with weighted centroid calculations. He et al. [2] propose APIT, where nodes decide position based on the possibility 
of being inside a triangle formed by three beacons. Global Coordinate System [4] uses a priori knowledge of node density to estimate average hop distance. The DV-* family of algorithms [18] infers distance to known beacons using hop counts. More recent research explores node positioning relative to events emanating from both predictable, known [19] and unpredictable, unknown [20] locations.

This paper extends previous work [21] that focused on design challenges in event-based localization. Different from previous work, we broaden and more precisely define various EDFs. Point Scan and Line Scan EDFs are applied to scenarios including unknown terrain, flat terrain, and known terrain. Area Cover EDF is further defined and optimal code placement is investigated. Hybrid solutions combining elements of Point Scan, Line Scan, and Area Cover EDFs are developed for scenarios that minimize localization error or time. Node density and distribution are also analyzed in these scenarios.

\section{Conclusions}

This paper presents the design, implementation, and evaluation of Spotlight, a localization system for wireless sensor networks. The 3D localization solution does not require any additional hardware on the sensor nodes. All system complexity is encapsulated into a single Spotlight device. The localization system is reusable and costs can be amortized through several deployments. Performance of the system is not affected by the number of sensor nodes in the network. Experimental results, obtained from a real system deployed outdoors, show that localization error is less than $20 \mathrm{~cm}$. This error is currently state of art, even for range-based localization systems, and is 75 percent smaller than the error obtained using GPS devices or when manual deployment of sensor nodes is a feasible option [22].

\section{ACKNOWLEDGMENTS}

A preliminary version of the paper was presented at the 2005 ACM Conference on Embedded Networked Sensor Systems (SenSys). This work was supported by US Defense Advanced Research Projects Agency (DARPA) grant F336616-01-C-1905 and US National Science Foundation (NSF) grants CCR-0098269, CNS-0626614, CNS-0923203.

\section{REFERENCES}

[1] N. Bulusu, J. Heidemann, and D. Estrin, "GPS-Less Low Cost Outdoor Localization for Very Small Devices," IEEE Personal Comm. Magazine, vol. 7, no. 5, pp. 28-34, Oct. 2000.

[2] T. He, C. Huang, B. Blum, J.A. Stankovic, and T. Abdelzaher, "Range-Free Localization Schemes in Large Scale Sensor Networks," Proc. ACM MobiCom, 2003.

[3] L. Hu and D. Evans, "Localization for Mobile Sensor Networks," Proc. ACM MobiCom, 2004.

[4] R. Nagpal, H. Shrobe, and J. Bachrach, “Organizing a Global Coordinate System from Local Information on an Ad Hoc Sensor Network," Proc. Second Int'l Conf. Information Processing in Sensor Networks (IPSN), 2003.

[5] B. Parkinson and J. Spilker, Global Positioning System: Theory and Applications, Progress in Aeronautics and Astronautics. Am. Inst. of Aeronautics, 1996.

[6] A. Savvides, C. Han, and M. Srivastava, "Dynamic Fine-Grained Localization in Ad-Hoc Networks of Sensors," Proc. ACM MobiCom, 2001.
[7] N. Priyantha, H. Balakrishnan, E. Demaine, and S. Teller, "MobileAssisted Topology Generation for Auto-Localization in Sensor Networks," Proc. IEEE INFOCOM, 2005.

[8] Diode Laser Modules and Systems, Coherent, Inc., 2005.

[9] N. Priyantha, A. Chakraborty, and H. Balakrishnan, "The Cricket Location-Support System," Proc. ACM MobiCom, 2000.

[10] P. Bahl and V.N. Padmanabhan, "Radar: An in-Building Rf-Based User Location and Tracking System," Proc. IEEE INFOCOM, 2000.

[11] K. Lorincz and M. Welsh, "Motetrack: A Robust Decentralized Approach to RF-Based Location Tracking," Proc. Int'l Workshop Location and Context-Awareness, 2005.

[12] P. Corke, R. Peterson, and D. Rus, "Networked Robots: Flying Robot Navigation Using a Sensor Net," Proc. Int'l Symp. Robotics Research (ISRR), 2003.

[13] P.N. Pathirana, A. Savkin, S. Jha, and N. Bulusu, "Node Localization Using Mobile Robots in Delay-Tolerant Sensor Networks," IEEE Trans. Mobile Computing, vol. 4, no. 3, pp. 285296, May/June 2005.

[14] M. Sichitiu and V. Ramadurai, "Localization of Wireless Sensor Networks with a Mobile Beacon," Proc. IEEE Int'l Conf. Mobile AdHoc and Sensor Systems, 2004.

[15] G. Simon, M. Maroti, A. Ledeczi, G. Balogh, B. Kusy, A. Nadas, G. Pap, J. Sallai, and K. Frampton, "Sensor Network-Based Countersniper System," Proc. Int'l Conf. Embedded Networked Sensor Systems (SenSys), 2004.

[16] A. Barton-Sweeney, D. Lymberopoulos, and A. Savvides, "Sensor Localization and Camera Calibration in Distributed Camera Sensor Networks," Proc. Third Int'l Conf. Broadband Comm. Networks and Systems (BROADNETS), pp. 1-10, 2006.

[17] S. Schuhmann, K. Herrmann, K. Rothermel, J. Blumenthal, and D. Timmermann, "Improved Weighted Centroid Localization in Smart Ubiquitous Environments," Proc. Int'l Conf. Ubiquitous Intelligence and Computing, pp. 20-34, 2008.

[18] D. Niculescu and B. Nath, "Ad-Hoc Positioning System," Proc. IEEE Global Telecomm. Conf. (GLOBECOM), 2001.

[19] Z. Zhong and T. He, "MSP: Multi-Sequence Positioning of Wireless Sensor Nodes," Proc. Int'l Conf. Embedded Networked Sensor Systems (SenSys), 2007.

[20] Z. Zhong, D. Wang, and T. He, "Sensor Node Localization Using Uncontrolled Events," Proc. Int'l Conf. Distributed Computing Systems (ICDCS), 2008.

[21] R. Stoleru, T. He, J.A. Stankovic, and D. Luebke, "A HighAccuracy Low-Cost Localization System for Wireless Sensor Networks," Proc. Int'l Conf. Embedded Networked Sensor Systems (ENSS), 2005.

[22] R. Stoleru, T. He, and J.A. Stankovic, "Walking GPS: A Practical Localization System for Manually Deployed Wireless Sensor Networks," Proc. 29th Ann. IEEE Int'l Conf. Local Area Networks, 2004.

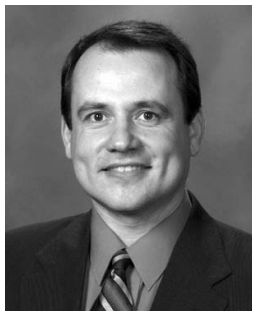

Radu Stoleru received the $\mathrm{PhD}$ degree in computer science from the University of Virginia in 2007, under Professor John A. Stankovic. He is currently an assistant professor in the Department of Computer Science and Engineering at Texas A\&M University. While at the University of Virginia, he received from the Department of Computer Science the Outstanding Graduate Student Research Award for 2007. His research interests are in deeply embedded wireless sensor systems, distributed systems, embedded computing, and computer networking. He has authored or coauthored more than 45 conference and journal papers with more than 1,800 citations. He is currently serving as an editorial board member for three international journal and has served as technical program committee member on numerous international conferences. He is a member of the IEEE and the ACM. 


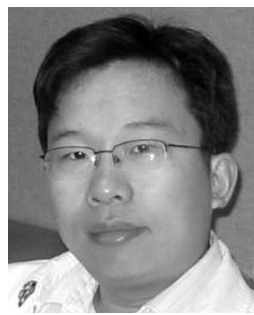

Tian He received the $\mathrm{PhD}$ degree under Professor John A. Stankovic from the University of Virginia in 2004. He is currently an associate professor in the Department of Computer Science and Engineering at the University of Minnesota-Twin City. He is the author and coauthor of more than 90 papers in premier sensor network journals and conferences with more than 4,000 citations. His publications have been selected as graduate-level course materials by more than 50 universities in the United States and other countries. $\mathrm{He}$ has received a number of research awards in the area of sensor networking, including four best paper awards (MSN 2006 and SASN 2006, MASS 2008, MDM 2009). He is also the recipient of the US National Science Foundation (NSF) CAREER Award 2009 and McKnight Land-Grant Professorship 2009-2011. He served a few program chair positions in international conferences and on many program committees, and also currently serves as an editorial board member for four international journals including ACM Transactions on Sensor Networks. His research includes wireless sensor networks, intelligent transportation systems, real-time embedded systems and distributed systems, supported by US National Science Foundation and other agencies. He is a member of the ACM and the IEEE.

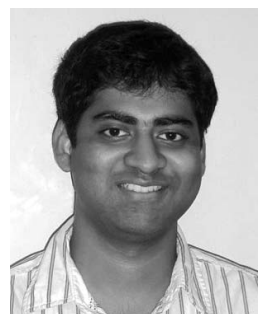

Siddhartha S. Mathiharan received the bachelor's degree in computer science from National Institute of Technology, Trichy, India, in 2008. He is currently working toward the master's degree in the Department of Computer Science at Texas A\&M University, College Station. His research interests include Computer Networks, Internet and distributed systems. $\mathrm{He}$ has authored several papers in international conferences.

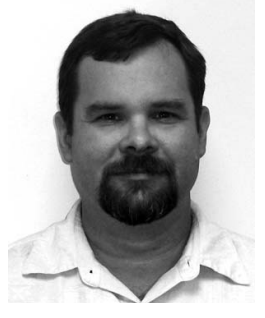

Stephen M. George received the MS degree in software engineering from Southern Methodist University. He is currently working toward the $\mathrm{PhD}$ degree in the Department of Computer Science and Engineering at Texas A\&M University. His research interests include security and adaptive behavior in large-scale wireless sensor networks, particularly in disaster response and military applications.

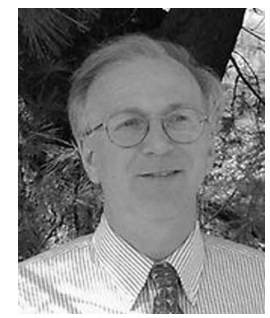

John A. Stankovic received the PhD degree from Brown University. He is the BP America professor in the Computer Science Department at the University of Virginia. In the past, he served as chair of the Department for eight years. He also won the IEEE Real-Time Systems Technical Committee's Award for Outstanding Technical Contributions and Leadership. He also won the IEEE Technical Committee on Distributed Processing's Distinguished Achievement Award (inaugural winner). He has won four Best Paper awards in sensor networks including for ACM SenSys 2006. Before joining the University of Virginia, he taught at the University of Massachusetts where he won an outstanding scholar award. He has also held visiting positions in the Computer Science Department at Carnegie-Mellon University, at INRIA in France, and Scuola Superiore $\mathrm{S}$. Anna in Pisa, Italy. He was the editor-in-chief for IEEE Transactions on Distributed and Parallel Systems and was founder and coeditor-inchief for the Real-Time Systems Journal. His research interests are in cyber physical systems, distributed computing, real-time systems, wireless sensor networks, and security for sensor networks. He is a fellow of both the IEEE and the ACM.

$\triangleright$ For more information on this or any other computing topic please visit our Digital Library at www.computer.org/publications/dlib. 